\title{
Exceso de confianza y optimismo en las decisiones de presupuesto de capital: las finanzas corporativas desde un enfoque centrado en el comportamiento
}

Overconfidence and Optimism in Capital Budget Decisions: Corporate Finances from a Behavioral Point of View

Excesso de confiança e otimismo nas decisões de pressuposto de capital: as finanças corporativas desde um enfoque centrado no comportamento

\section{Alejandro José Useche Arévalo*}

Fecha de recibido: 11 de febrero de 2013

Fecha de aprobado: 7 de julio de 2013

Doi: dx.doi.org/10.12804/rev.univ.empresa.26.2014.03

Para citar este artículo: Useche A., A. J. (2014). Exceso de confianza y optimismo en las decisiones de presupuesto de capital: las finanzas corporativas desde un enfoque centrado en el comportamiento. Universidad \& Empresa, 16(26), 95-1 16. doi: dx.doi.org/10.12804/rev.univ.empresa.26.2014.03

\section{RESUMEN}

Este artículo presenta, como resultado de la revisión de literatura efectuada como un proyecto de investigación, un análisis comparativo entre el proceso de evaluación de decisiones de inversión, desde la teoría financiera tradicional, y un nuevo enfoque complementario que surge desde las finanzas corporativas basadas en el comportamiento (Behavioral Corporate Finance), un campo creciente de investigación en el cual se incorporan las influencias emocionales y cognitivas con el fin de lograr una mejor comprensión respecto a la toma de decisiones empresariales. Se analiza, en particular, la forma en la que los sesgos en el comportamiento, denominados exceso de confianza y optimismo, influyen de forma sistemática

Doctor of Business Administration (DBA) de la Swiss Management Center University. Profesor de carrera, Universidad del Rosario. Correo electrónico: alejandro.useche@urosario.edu.co 
en el proceso de presupuesto de capital. La evidencia empírica demuestra que dichos sesgos conducen a sobreestimar los flujos de caja netos esperados y a subestimar sus varianzas, lo cual resulta principalmente en decisiones financieras sub-óptimas (i.e. sobreinversión y excesiva exposición al riesgo), aunque en ocasiones originan también prácticas que aportan valor a la empresa (i.e. rapidez en la ejecución de proyectos y mayor compromiso). El trabajo plantea recomendaciones para corregir las distorsiones que los sesgos psicológicos del exceso de confianza y el optimismo generan sobre el proceso de presupuesto de capital. Palabras clave: Exceso de confianza, finanzas corporativas basadas en el comportamiento, optimismo, sesgos psicológicos, toma de decisiones financieras.

\section{ABSTRACT}

As result of the literature review carried out as a research project, this article presents a comparative analysis of the evaluation process of investment decisions from the viewpoint of the traditional financial theory and a new complementary approach that arises from Behavioral Corporate Finance, a growing research field which incorporates emotional and cognitive influences with the purpose of obtaining better understanding with respect to corporate decision making. In particular, we analyze the way that behavioral biases, called overconfidence and optimism, systemically influence the process of capital budgeting. Empirical evidence shows that these biases lead to overestimating the expected net cash flows and underestimating their variances, resulting mainly in sub-optimal financial decisions (i.e. overinvestment and excessive exposure to risk.), although they occasionally create practices that add value to the company (i.e. speed in the execution of projects and greater commitment). The paper makes recommendations aimed at correcting distortions that psychological biases of overconfidence and optimism generate on the capital budgeting process.

Keywords: Corporate behavioral finance, Financial decision making, Optimism, Overconfidence, Psychological bias.

\section{RESUMO}

Este artigo apresenta, como resultado da revisão de literatura efetuada como um projeto de pesquisa, uma análise comparativa entre o processo de avaliação de decisões de inversão, desde a teoria financeira tradicional, e uma nova abordagem complementária que surge desde as Finanças Corporativas baseadas no Comportamento (Behavioral Corporate Finance), um campo crescente de pesquisa no qual se incorporam as influências emocionais e cognitivas com o fim de conseguir uma melhor compreensão respeito à tomada de decisões empresariais. Analisa-se, em particular, a forma na que os vieses no comportamento, denominados excesso de confiança e optimismo, influem de forma sistemática no processo de pressuposto capital. A evidência empírica demonstra que ditos vieses levam a sobrestimar os fluxos de caixa netos esperados e a subestimar suas varianças, o qual resulta principalmente em decisões financeiras subótimas (i.e. sobreinvestimento e excessiva 
exposição ao risco), ainda que em ocasiões originam também práticas que aportam valor à empresa (i.e. rapidez na execução de projetos e maior compromisso). O trabalho expõe recomendações para corrigir as distorções que es vieses psicológicos do excesso de confiança e o optimismo geram sobre o processo de pressuposto de capital.

Palavras-chave: Excesso de confiança, finanças corporativas baseadas no comportamento, otimismo, tomada de decisões financeiras, vieses psicológicos.

\section{INTRODUCCIÓN}

Las decisiones de presupuesto de capital se encuentran dentro de las que generan mayores impactos sobre el desempeño de largo plazo de las organizaciones. Tales decisiones se basan principalmente en proyecciones y en el pronóstico de posibles comportamientos futuros del negocio y su entorno, variables que en buena medida no están bajo el control de la empresa ni son predecibles con precisión (Schönbohm $\&$ Zahn, 2012). Frente a este proceso de toma de decisión bajo incertidumbre, el enfoque tradicional de las finanzas corporativas y en particular del análisis de las decisiones de presupuesto de capital se basa en supuestos según los cuales los agentes pueden ser calificados no solo como "racionales" (en el sentido de ser lógicos en sus procesos de elección), sino más aún como "hiperracionales", ya que se supone que toman siempre decisiones optimizadoras, cuentan con preferencias claramente definidas, no tienen curvas de aprendizaje, conocen el conjunto de resultados posibles - los cuales a su vez son fijos- - y escogen de manera inequívoca aquella alternativa que genera el mayor beneficio (Baker \& Wurgler, 2013; Fairchild, 2009; Jones, 1999).

Dicho supuesto de la hiper-racionalidad de los agentes individuales fue extendido al conjunto de los mercados financieros por medio de la llamada Hipótesis de los Mercados Eficientes, término creado por Harry Roberts en 1967 (Shiller, 1999) y difundido por Eugene Fama como una característica sobre el comportamiento del mercado de acciones, que con el tiempo se hizo extensivo a otros activos financieros (Fox, 2010). Así, en la literatura tradicional de las finanzas corporativas los directivos dan por hecho que los mercados financieros son eficientes y a su vez los inversionistas suponen que los directivos toman siempre decisiones óptimas, técnicamente calculadas, orientadas a la maximización del valor de la empresa (Shah, 2013).

En el caso particular de las decisiones de inversión en activos fijos, es 
común la utilización de métodos como el valor presente neto (VPN) y la tasa interna de retorno (TIR), los cuales a pesar de su consistencia matemática pueden estar influenciados por elementos subjetivos que llevan, por ejemplo, a sobreestimar los ingresos de un proyecto y a subestimar sus costos, generando conclusiones imprecisas que resultan en decisiones de presupuesto de capital inadecuadas y altamente riesgosas.

Las "finanzas corporativas basadas en el comportamiento (FCBC)" o Behavioral Corporate Finance (BCF) son un enfoque innovador en el que se reemplazan los supuestos mencionados de híper-racionalidad por principios basados en la observación de la conducta real de los empresarios, la cual está sujeta a juicios de valor y a errores propios de la naturaleza humana. En la práctica, la psicología ha demostrado que los procesos de toma de decisiones son altamente influenciados por elementos como los heurísticos, definidos como formas de "buscar la solución de un problema mediante métodos no rigurosos, como por tanteo, reglas empíricas, etc.”. (Real Academia Española [RAE], 2013).

Así, mediante la experiencia acumulada por el ensayo y error, las personas van encontrando principios que consideran de forma per- sonal como reglas generales, pero, en ocasiones, tal proceso lleva a errores que se convierten en conductas sistemáticas. Howard y Yazdipour (2010) complementan esta descripción afirmando que:

Cuando se enfrentan a grandes cantidades de datos e información y un despliegue de problemas a solucionar, las personas son incapaces de hacer los complejos cálculos de optimización que se espera de ellas bajo la teoría financiera estándar. En vez de eso, confían en un número limitado de estrategias cognitivas o heurísticos que simplifican los complejos escenarios a que se enfrentan cuando toman decisiones. Los heurísticos son atajos en el procesamiento de información que resultan principalmente de la experiencia propia en un campo de trabajo. Por supuesto, tales atajos simplificadores son productivos y les permiten a los humanos funcionar en la vida diaria. Por naturaleza, los heurísticos son imperfectos y consecuentemente resultarán en desviaciones y errores (p. 45).

El ganador del premio Nobel de economía 2002, Daniel Kahneman (1998) concluye entonces que "los inversionistas que son propensos a estos sesgos tomarán riesgos que no reconocen, obtendrán resultados que no anticipan, serán propensos a transacciones injustificadas, y podrán terminar culpándose a ellos 
mismo o a otros cuando los resultados sean malos" (p. 53). Como un aporte a este debate, el presente artículo ofrece una descripción de los principales aspectos que las $\mathrm{FCBC}$ han desarrollado para la comprensión de la influencia de los sesgos de optimismo y exceso de confianza en las decisiones de presupuesto de capital en las empresas, al tiempo que presenta una revisión de la literatura más relevante al respecto.

\section{ENFOQUE TRADICIONAL DEL PROCESO DE PRESUPUESTO DE CAPITAL}

La literatura identifica tradicionalmente tres decisiones principales del área financiera de las organizaciones: la administración del capital de trabajo, la estructura de capital y el presupuesto de capital (Ehrhardt y Brigham, 2011). Esta última labor está relacionada con la evaluación, selección y ejecución de inversiones de largo plazo de la compañía. El proceso de presupuesto de capital implica la necesidad de cuantificar las inversiones y estimar los montos y los momentos en que los ingresos tendrán lugar. Un proceso que tiene un impacto directo en decisiones trascendentales como la de iniciar o no un nuevo proyecto, ampliar un área ya existente, adquirir activos de capital, reasignar recursos entre las diferentes áreas de la empresa, o llevar a cabo procesos de fusiones o adquisiciones, entre otros.

El enfoque más extendido en la teoría financiera y en la práctica empresarial es el de definir detalladamente los ingresos y los egresos relacionados con el proyecto, seleccionar una vida útil u horizonte de tiempo, proyectar los flujos de caja esperados y, dada una tasa de interés de descuento que refleje el costo de capital y el riesgo, calcular indicadores como el valor presente neto y la tasa interna de retorno, de forma que sea posible concluir si el proyecto será viable o no desde el punto de vista financiero, para finalmente aprobar la inversión si se considera que ella contribuirá al objetivo de maximización de valor para los accionistas (Axelsson et al., 2002; Fornero, 2010).

Formalmente, la empresa estima cada uno de sus flujos de caja netos (FCNt) y los proyecta a valor presente usando una tasa de descuento $r$ (que en un escenario básico se supone constante en el tiempo), para hallar el valor presente neto (vPN) así:

$$
\begin{aligned}
& V P N=F C N_{o}+\frac{F C N_{1}}{1+r}+\frac{F C N_{2}}{(1+r)^{2}}+\ldots+\frac{F C N_{\mathrm{n}}}{(1+r)^{n}} \\
& V P N=\sum_{t=0}^{n} \frac{F C N_{t}}{(1+r)^{t}}
\end{aligned}
$$


Dado que el VPN es una medida relativa de la cantidad de dinero equivalente en el momento $t=0$, que se espera el proyecto generará por encima de la tasa de descuento $r$, la empresa decidirá invertir cuando $\mathrm{VPN}>0$, o no invertir si VPN $<0$. Tomar la decisión de ejecutar un proyecto con VPN positivo incrementará la riqueza de la empresa y creará valor (Stowe \& Gagné, 2012).

Por su parte la tasa interna de retorno (TIR) es aquella tasa de descuento que hace que el VPN sea igual a cero, siendo una aproximación de la rentabilidad de los recursos invertidos en el proyecto (bajo el supuesto de que todos los flujos de caja que se liberan son reinvertidos en él, bajo las mismas condiciones inicialmente proyectadas). Siendo $\mathrm{I}_{0}$ el valor de la inversión inicial, la TIR es entonces la tasa de descuento que equilibra los flujos de caja, de la siguiente forma:

$$
\sum_{t=1}^{\mathrm{n}} \frac{F C_{t}}{(1+T I R)^{t}}-I_{0}=0
$$

De manera similar al VPN, el criterio de evaluación es que será conviene para la empresa invertir si la TIR es mayor que la tasa mínima de rentabilidad deseada del proyecto, lo que implica a la vez un VPN positivo. Este enfoque básico puede ser com- plementado, por ejemplo, mediante la proyección de varios escenarios y la definición de probabilidades para cada uno, o mediante métodos que implican la inclusión de elementos aleatorios, bajo enfoques como las opciones reales o la simulación de Montecarlo.

La regla del valor presente neto propuesta por primera vez de manera formal en las finanzas corporativas por Dean (1951) — sigue siendo el principal criterio para la toma de decisiones de presupuesto de capital. Este es un hecho que autores como Graham y Harvey (2001) han documentado ampliamente. Harris, Emmanuel y Komakech (2009) afirman al respecto que el método del valor presente neto:

Es un modelo estático, que falla en reflejar la naturaleza dinámica de los negocios y la tendencia humana a formar juicios sobre la deseabilidad de un proyecto [...] El problema de proyectar flujos de caja futuros y resultados no monetarios implica que la toma de decisiones estratégicas de inversión no es una ciencia exacta, sino un ejercicio de estimación, abierto al error y a la imprecisión en los cálculos. En el intento de eliminar la incertidumbre, los directivos recurren naturalmente a su propia experiencia pasada en eventos similares y a su pericia profesional (p. 2). 
Un aspecto adicional es el hecho de que, aunque los mismos principios del presupuesto de capital sean estudiados alrededor del mundo, los aspectos socioculturales influyen en la conducta de las personas que toman las decisiones de inversión, de forma que los supuestos y las proyecciones realizadas varían sensiblemente de una cultura a otra (Statman, 2008).

\section{LAS FINANZAS CORPORATIVAS BASADAS EN EL COMPORTAMIENTO (FCBC)}

Las FCBC, un campo dedicado al estudio de la forma en que los elementos psicológicos influyen de manera sistemática en la toma de decisiones financieras, surgieron como una reacción a las diferencias entre el comportamiento observado de los agentes en los mercados financieros y las conductas que la teoría tradicional (principalmente expresada en el paradigma neoclásico) formula en términos normativos.

Recientemente este enfoque se ha extendido a campos específicos de las finanzas como las decisiones de inversión, la estructura de capital y la política de dividendos (Fairchild, 2009). Este hecho permitió la configuración de una nueva área denominada "finanzas corporativas basadas en el comportamiento" o
Behavioral Corporate Finance. Esta área puede complementar a la teoría tradicional aportando una comprensión más realista y humana de la forma en que se llevan a cabo en la práctica los procesos de asignación de recursos financieros en la empresa.

Los trabajos de Herbert Simon (premio Nobel de economía en 1978) en 1955 y de Julius Margolis en 1958 fueron los pioneros en reconocer de forma explícita la necesidad de que la teoría de la empresa fuera alimentada por los desarrollos de la psicología (Gervais, 2010). Sin embargo, solo hasta años recientes se ha desarrollado formalmente este enfoque alternativo en el cual se asume que los directivos son "racionales" en la toma de decisiones pero no cuentan con la capacidad ni con la información —o a veces con la voluntad, según Mattson y Weibull (2002) necesarias para conocer la distribución real de las variables que evalúan. Este es aspecto denominado "incertidumbre estructural" o "racionalidad acotada". Gracias a él se reconoce que aunque la toma de decisiones empresariales sea realizada por directivos profesionales, ellos no dejan de ser humanos, con limitaciones en el manejo de la información. Los directivos son individuos influenciados por elementos subjetivos, preferencias, creencias, intuición, presiones externas, esta- 
dos de ánimo, costumbres, variables que pueden llevar a que la decisión final diste de la recomendada por los modelos, generando una grieta entre la teoría y la práctica financieras (Graham y Harvey, 2001).

Según Damasio (1994) buena parte de los científicos ha llegado a creer que las buenas decisiones son tomadas solo por personas altamente racionales y con "cabeza fría", quienes para llegar a soluciones óptimas no permiten que sus emociones interfieran con sus razonamientos. Sin embargo, cada vez más resultados de procesos de investigación concluyen que para entender realmente el proceso de toma de decisiones económicas es necesario incluir elementos emocionales y cognitivos. Estos concluyen además que la ausencia de emociones puede ser en la práctica una gran fuente de comportamientos sub-óptimos, que no corresponden con los postulados de la racionalidad en el sentido neoclásico (Damasio, 1994; Kida, Moreno, \& Smith, 2001; LeDoux, 1993, 1996).

Shefrin (1999), uno de los más importantes autores de la escuela del comportamiento, agrupa los diferentes fenómenos psicológicos que afectan a las decisiones financieras en tres categorías, a saber: a) sesgos (biases): predisposiciones a cometer errores, $b$ ) heurísticos: atajos mentales para facilitar la toma de decisiones y $c$ ) efectos de encuadre (framing effects): cambios de decisión por la forma en que se presenta la información.

Investigaciones recientes (Biondi \& Marzo, 2011; Hribar \& Yang, 2010, 2012) han demostrado que algunos de los principales sesgos que afectan el proceso de presupuesto de capital en las empresas son los denominados "optimismo" (optimism), consistente en la sobreestimación de los flujos de caja netos esperados, y el "exceso de confianza" (overconfidence), una tendencia por la cual se estima una menor varianza de tales flujos decaja netos.

Así, mientras la literatura tradicional se ha concentrado en las técnicas para valorar los flujos de caja, poca atención se ha prestado a desarrollar modelos que reflejen los métodos que en la práctica usan los directivos para tomar sus decisiones de inversión de largo plazo, y a estimar los efectos que influencias emocionales y cognitivas generan en la toma de decisiones financieras empresariales.

\section{ENFOQUE DEL COMPORTA- MIENTO EN EL PRESUPUESTO DE CAPITAL}

Los trabajos de investigación en el área de las FCBC han encontrado 
evidencia de la influencia sistemática de aspectos subjetivos en la toma de decisiones corporativas. Avances recientes como los de Ben-David, Graham y Harvey (2010), así como los de Sautner y Weber (2009) hallaron evidencia empírica de la forma en que la toma de decisiones sobre la adquisición de activos fijos $\mathrm{y}$, en general, sobre la evaluación de la viabilidad de un proyecto de inversión de largo plazo, suele estar influenciada en el alto nivel directivo por los sesgos de optimismo y exceso de confianza. En el mismo sentido, Heaton (2002) afirma que el exceso de confianza lleva a los directivos a sobreestimar el VPN de nuevos proyectos, haciendo que inviertan en alternativas que muy probablemente tendrán VPN negativo. Esto genera pérdidas y destruye valor para los accionistas.

Por otro lado, mientras se desarrollan en la teoría métodos cada vez más sofisticados para la toma de decisiones financieras en la empresa, en la práctica tales decisiones son tomadas en muchas ocasiones a través de procedimientos simples, más comprensibles y con los que el directivo a cargo tiene un mayor grado de familiaridad. Una situación en la que los sesgos, los heurísticos y los efectos de encuadre juegan un papel importante. Estudios como los realizados por Burns y Walker (1997) y por Biondi y Marzo (2011) muestran que la facilidad de recolección de información y de cálculo juega un papel primordial al momento de seleccionar el método para realizar el presupuesto de capital.

Entre un amplio conjunto de heurísticos documentado en la literatura, el presente documento se concentrará en analizar la influencia del "exceso de confianza" y del "optimismo" en el proceso de presupuesto de capital.

\section{A. Exceso de confianza}

El exceso de confianza (overconfidence) es una tendencia por la cual las personas suelen creer que sus habilidades para ciertas actividades son mayores que las del individuo común. Basta con preguntar a un grupo de personas cuántas de ellas piensan que conducen un automóvil mejor que el conductor promedio o, en un caso más dramático, cuántas de ellas piensan que tienen una esperanza de vida mayor que el promedio. Al respecto pueden consultarse los trabajos de Weinstein (1980) y Svenson (1981).

Diversos estudios han mostrado que en una amplia gama de actividades de negocios los individuos tienden a sobreestimar la precisión de la información con 
que cuentan, de sus conocimientos y de sus habilidades. Este es un comportamiento encontrado en especial en directivos que cuentan con una larga trayectoria (Camerer \& Lovallo, 1999; Gervais, Heaton, \& Odean, 2011; Russo \& Schoemaker, 1992). Esta tendencia lleva a que la empresa invierta más recursos de lo debido y se exponga más ampliamente a los riesgos, lo que puede resultar en desempeños extremadamente buenos o sumamente malos (Baker \& Wurgler, 2013).

Los resultados de los estudios empíricos han arrojado evidencia en favor de la presencia de exceso de confianza en buena parte de las decisiones ejecutivas. Se ha verificado, por ejemplo, que empleados con exceso de confianza en sus habilidades tienen mayor probabilidad de ganar convocatorias internas para ocupar altos cargos directivos como Chief Executive Officer (CEO) (Goely Thakor, 2008). Adicionalmente, ejecutivos exitosos suelen atribuir gran parte de sus logros a sus propias habilidades, al tiempo que atribuyen sus fracasos a la mala suerte, lo que reafirma su exceso de confianza (Daniel, Hirshleifer, \& Subrahmanyam, 1998; Gervais \& Odean, 2001).

Autores como Graham y Harvey (2001) encuentran que las compañías con directivos que tienen de exceso de confianza tienden a usar menores tasas de descuento para la evaluación de sus proyectos. Ellos tienden a financiarlos con mayores niveles de deuda, la que en su mayoría es de largo plazo (Malmendier \& Tate, 2005). Un aspecto interesante a resaltar es que los emprendedores muestran excesos de confianza superiores al promedio. Koellinger, Minniti y Schade (2007) hallaron que las percepciones subjetivas y frecuentemente sesgadas de los emprendedores tienen un impacto profundo en su decisión de creación de empresa, una conclusión que contó con evidencia robusta en los países que compusieron su muestra. Adicionalmente, hubo una correlación directa entre las características socioculturales del entorno y los niveles de confianza en el desempeño futuro, pero se presentó una correlación inversa entre tal exceso de confianza y las probabilidades de supervivencia de las nuevas empresas.

Un posible signo de la perdurabilidad de este heurístico es que los emprendedores, al parecer, no reducen de forma significativa su optimismo como resultado de fracasos anteriores. Ellos reinciden en la realización de proyecciones sobreestimadas de sus flujos de caja y de la viabilidad de sus inversiones (Ucbasaran, Westhead, Wright, \& Flores, 2010). 
Landier y Thesmar (2009) encontraron que, en el caso de Francia, los emprendedores subestiman ampliamente las posibilidades de fracasar, pues solo el $6 \%$ de los encuestados consideró que su negocio podría pasar por dificultades, una idea que se traslada a la forma en que definen sus flujos de caja y evalúan sus proyectos de inversión. Sin embargo, las estadísticas mostraron que en promedio solo la mitad de las empresas nuevas sobrevivió luego de los tres años iniciales de funcionamiento (Scarpetta, Hemmings, Tressel, \& Woo, 2002).

En términos de la decisión sobre el presupuesto de capital, el exceso de confianza se presenta principalmente en las etapas de formulación y evaluación de un proyecto, de forma que los directivos sobreinvierten recursos, particularmente en casos en que dicho proyecto se financia a partir de los propios flujos de caja de la empresa. Además, existe una tendencia por la cual los directivos proyectan una varianza menor de los flujos de caja netos que en la práctica son procesos aleatorios-, definiendo intervalos de confianza demasiado estrechos o incluso haciendo pronósticos puntuales (point forecasts). Esto conduce a aumentar la probabilidad de fallar en las proyecciones (Hribar \& Yang, 2010).
Un aspecto positivo del exceso de confianza es que, como han argumentado Gervais (2010) y Brealey, Myers y Allen (2011), esta conducta tiende a contribuir al logro de las ambiciosas metas propuestas en el proyecto. Esto debido a un mayor nivel de compromiso de las directivas, lo que constituye una profecía autocumplida. Sin embargo, un grave problema, que resulta de llevar a cabo proyecciones en exceso optimistas, es el de caer en la tentación de realizar fraude para, de forma manipulada, alcanzar o superar unas metas excesivas. En este sentido, Schrand y Zechman (2011) encontraron una fuerte relación entre los niveles de exceso de confianza por parte de los ejecutivos y los fraudes corporativos.

\section{B. Optimismo}

Otro de los heurísticos que tiene influencia sobre el proceso de presupuesto de capital es el optimismo, que puede ser definido como una tendencia psicológica por la cual los agentes sobreestiman la posibilidad de disfrutar de escenarios futuros positivos (Baker \& Wurgler, 2013). En relación con el presupuesto de capital, el optimismo lleva a sobreestimar los ingresos esperados y a subestimar los egresos esperados, generando un sesgo de las proyecciones de los diferentes indicadores 
financieros de evaluación de los proyectos.

Diversos estudios, desde Pohlman, Santiago y Markel (1988), han tratado de verificar empíricamente el efecto del optimismo en las proyecciones de las empresas. Estos autores, en particular, realizaron una encuesta en las empresas del listado de Fortune 500 y descubrieron diferencias significativas entre los flujos de caja proyectados y los efectivamente causados, diferencias que, en buena medida, se explicaron por la influencia de aspectos subjetivos propios de la experiencia o la personalidad de los directivos de las compañías.

En este orden de ideas, no es difícil ver cómo en la práctica las decisiones empresariales, incluyendo las relacionadas con presupuesto de capital, más que aplicar ciegamente las recomendaciones de los modelos teóricos, son el resultado de la orientación que el equipo directivo quiere darle a la organización, reflejando aspectos como la personalidad de sus miembros. Evidencia de esto fue dada por Schoar y Bertrand (2003), quienes demostraron el "efecto CEO" en las decisiones sobre estructura de capital. Ellos pusieron en evidencia cómo las decisiones ejecutivas de las empresas analizadas reflejaron el estilo per- sonal de sus directivos más que los criterios generales definidos por la organización. Por ejemplo, empresas que tienen directivos con estilos conservadores presentan mayores niveles de efectivo en sus balances y al momento de definir sus fuentes de crecimiento prefieren hacer uso de recursos internos, mientras que empresas con directivos de estilos más agresivos - generalmente ejecutivos jóvenes (Adler, 2004)conservan menos efectivo, están en promedio más apalancadas y tienden a optar por adquisiciones para su crecimiento, exponiendo a la empresa a mayores riesgos, pero obteniendo en promedio mejores rentabilidades sobre el capital. Más recientemente, Cronqvist, Makhija y Yonker (2012) encontraron una relación robusta entre las decisiones corporativas de endeudamiento de una empresa y las decisiones de endeudamiento personales de su CEO.

Igual que en el caso del exceso de confianza, el sesgo de optimismo puede en ocasiones generar beneficios para la empresa, no solamente el directivo optimista tiende a ser una persona "más feliz, más popular, más dada a ayudar a los demás y más dispuesta a persistir en sus tareas" (Gervais, Heaton, \& Odean, 2002 , p. 26), lo que propicia un mejor clima organizacional, sino que puede incrementar el valor de la 
compañía, ya que, a diferencia de los directivos excesivamente prudentes, los ejecutivos optimistas son menos propensos a posponer las decisiones de inversión y, por el contrario, inician los proyectos rápidamente, al tiempo que dedican más esfuerzos a ellos para obtener los resultados proyectados (Felleg, Moers, \& Renders, 2012).

Otra prueba de los efectos del optimismo se encuentra en los casos en que los directivos de las empresas se rehúsan a abandonar un proyecto en marcha, aun cuando los cálculos ajustados del VPN así lo recomienden: el optimismo de que los flujos de caja netos serán mejores que los proyectados (y el temor de perder las inversiones ya hechas) lleva a aferrarse a inversiones que los indicadores muestran como inconvenientes (Fairchild, 2004; Statman \& Caldwell, 1987).

Se ha encontrado también que las personas tienden a ser más optimistas acerca de los resultados que creen tener bajo control, así como con aquellos provenientes de tareas con las que se encuentran altamente comprometidas. Heaton (2002) provee evidencia empírica al respecto, según la cual los directivos no solo "parecen estar generalmente comprometidos con el éxito de la organización", sino que "creen poseer altos niveles de control sobre el desempeño de la empresa" (p. 33). Por último, Shefrin y Cervellati (2011) muestran cómo un optimismo excesivo no solamente puede perjudicar los intereses de la empresa, sino acarrear terribles daños a terceros. Tal es el caso del desastre natural producido por el derrame de petróleo en el Golfo de México en abril de 2010, en el que los ejecutivos de la British Petroleum (BP), a cargo del proyecto, parecen haber subestimado la posibilidad de ocurrencia de hechos adversos. Por ello, realizaron cortes en gastos de mantenimiento y proyectaron inversiones muy bajas en seguridad. Más aún, el exagerado optimismo se mantuvo en pleno desarrollo de la crisis, cuando en sus declaraciones públicas minimizaron repetidamente el verdadero impacto de la tragedia que estaba ocurriendo (Crooks, 2010).

\section{UN MODELO BÁSICO DE PRESUPUESTO DE CAPITAL CENTRADO EN EL COMPORTAMIENTO}

En esta sección se presenta un modelo desarrollado por Gervais (2010) y Gervais, Heaton y Odean (2011) - con algunos ajustes hechos por el autor de este artículo- en el que se considera la decisión de presupuesto de capital desde un enfoque de FCBC. En él los sesgos de exceso 
de confianza y optimismo son incluidos en la decisión de presupuesto de capital. Se supone una empresa que tiene un horizonte de proyección de un solo periodo, con un agente al que se denominará directivo, responsable del proceso de selección de inversiones de largo plazo, que tiene como objetivo maximizar el valor corporativo, es decir, que está libre de problemas de agencia.

La empresa debe incurrir en una inversión inicial hoy por un monto conocido de I $>0$, a cambio de la cual obtendrá un flujo de caja $\widetilde{F C}$ al final del periodo (monto desconocido en el momento inicial), donde $\widetilde{F C}$ es una variable aleatoria con media $\overline{F C}$ y que asume valores entre $(-\infty, \infty)$. El directivo elige una tasa de descuento $r$, que refleja el costo de capital y el riesgo del proyecto, y toma la decisión de realizarlo o no, la cual se expresa en la variable dicotómica $D$. Ella asume el valor de 1 en el caso en que se decide hacer el proyecto, o de 0 en caso contrario. De esta forma, el VPN toma la forma de una variable aleatoria, expresada de la siguiente manera:

$$
\widetilde{V P N}=D\left(\frac{F C}{1+r}-I\right)
$$

Se supone ahora que es posible que el directivo cuente con la habilidad para obtener e interpretar información adicional no observable para la empresa en general, sobre los posibles ingresos que generará el proyecto que está evaluando, de forma que usando su buen juicio pueda tener más elementos para tomar una decisión de inversión que genere valor. Gervais denota como $\widetilde{s}$ a esta información, dándole el nombre de señal (signal), que se expresa como un promedio ponderado entre el valor que realmente tendrá el flujo de caja en el periodo 1 y otro posible valor $\widetilde{\psi}$, que tiene la misma distribución que $\widetilde{F C}$, pero que es independiente de él:

$$
\tilde{S}=\widetilde{\varepsilon} \overline{F C}+(1-\widetilde{\varepsilon}) \widetilde{\psi}
$$

Donde $\widetilde{\psi}$ es una variable dicotómica - no observable - que asume el valor de 1 con probabilidad $\alpha$, y de 0 con probabilidad $(1-\alpha)$, siendo el parámetro $\alpha \in\left[0, \frac{1}{2}\right]$ la posibilidad de que $\widetilde{s}$ resulte ser igual que $\widetilde{F C}$. En otras palabras, $\alpha$ expresa la verdadera habilidad del directivo (no la que él percibe de sí mismo). Lo anterior implica que:

$$
\begin{array}{r}
\mathrm{E}(\overline{\mathrm{FC}} \mid \widetilde{\mathrm{s}})=\alpha \widetilde{\mathrm{s}}+(1-\alpha) \overline{F C}= \\
\overline{F C}+\alpha(\mathrm{s}-\overline{F C})
\end{array}
$$

La expresión (5) implica que una señal $\widetilde{s}$ positiva llevará a aumentar el valor esperado del flujo de caja que 
realmente se obtendrá, de forma que el directivo proyectará unos ingresos mayores que el promedio $\overline{F C}$. Ahora bien, si el directivo presenta el sesgo de exceso de confianza, sobreestimará sus propias habilidades, lo cual implica en el modelo que a su verdadera habilidad $\alpha$ sumará un valor adicional $\beta \in\left[0, \frac{1}{2}\right]$, de forma que la ecuación (5) se modificará de la siguiente forma:

$$
\begin{gathered}
E_{\beta}(\overline{\mathrm{FC}} \mid \widetilde{s})=(\alpha+\beta) \widetilde{s}+(1-\alpha-\beta) \overline{F C}= \\
\overline{F C}+(\alpha+\beta)(\widetilde{s}-\overline{F C})
\end{gathered}
$$

En este caso, el directivo sobreestima el valor esperado del flujo de caja cuando $\tilde{S}>\overline{F C}$. Un coeficiente $\beta$ más alto significa que el directivo da una importancia mayor a su propia información, mientras que si $\beta$ $=0$ el directivo está libre de exceso de confianza y hace un cálculo insesgado de la información contenida en $\widetilde{s}$. El directivo decidirá desarrollar el proyecto de inversión cuando el valor esperado del VPN sea positivo, lo cual en este caso sucede si $\frac{E_{\beta}(\overline{\mathrm{FC}} \mid \Im)}{1+r}>I$. La presencia de un parámetro $\beta>0$ hace que se sobreestime el valor esperado del flujo de caja y, por lo tanto, que se tienda a proyectar un VPN alto, proceso que resulta en una inversión mayor que la debida.

Por su parte, el optimismo es incluido como la percepción de que se obten- drá un flujo de caja mayor que el definido por la distribución inicial, de forma que la media aumenta a $\overline{F C}$ $+\lambda$. El directivo optimista sobreestima el valor esperado de los flujos de caja del proyecto, así:

$E_{\lambda}(\overline{F C} \mid \widetilde{s})=\alpha \widetilde{s}+(1-\alpha)(\overline{F C}+\lambda)$

Gervais concluye que, en este escenario, el proyecto será considerado viable y se desarrollará si $\widetilde{F C}>F C_{\lambda}^{*}$, donde este último término se define como:

$F C_{\lambda}^{*}=F C+\lambda-\frac{F C+\lambda-I(1+r)}{\alpha}$

En un primer momento, el sesgo de optimismo lleva a sobreestimar el flujo de caja en el monto $\lambda$. Sin embargo, tal efecto puede ser reducido en la medida en que la habilidad real del directivo $(\alpha)$ sea mayor.

\section{RECOMENDACIONES PARA LA EMPRESA}

Como se presentó anteriormente, la evidencia muestra que las diferentes etapas del proceso de presupuesto de capital están sujetas a sesgos tales como el optimismo y el exceso de confianza, sesgos que llevan a sobreestimar los flujos de caja netos esperados de un proyecto. Para corregir tales distorsiones, no es suficiente (ni conveniente) utilizar una mayor tasa de descuento por el sim- 
ple hecho de que matemáticamente se reduciría de inmediato el VPN.

Si se acepta que, como afirmó Antonio Caso, estamos en un mundo "imperfecto pero perfectible" (Krauze, 1990 , p. 75), un primer paso para evitar los impactos negativos de los sesgos del comportamiento es el de reconocer que existen e identificar cómo ellos afectan a la toma de decisiones. Muy recientemente Schönbohm y Zahn (2012) afirmaron al respecto que:

Estar conscientes de las trampas de los diferentes sesgos subjetivos provee un cierto nivel de reflexión autocrítica, el cual finalmente podría llevar a proyecciones más razonables. Algunos directivos mejorarían sus habilidades para la toma de decisiones simplemente con crear conciencia de los sesgos psicológicos (p. 22).

En casos en que se toman decisiones financieras que intencionalmente destruyen valor (como aquellas que surgen por problemas de agencia), la literatura recomienda corregir el sistema de incentivos. Sin embargo, cuando los directivos destruyen valor de forma no intencional como consecuencia de sesgos psicológicos, probablemente la manera más adecuada de empezar a corregir el problema es mediante la educación y el entrenamiento (Fairchild,
2009). De esta forma, es posible desarrollar programas de capacitación primero a nivel de la alta dirección y luego, con su apoyo, con el equipo financiero encargado de la realización de las proyecciones. Estos programas no solo estarán orientados a reconocer los inconvenientes causados por los sesgos psicológicos, sino también a comprender la forma en que pueden ser aprovechados para la creación de valor. Es claro que una labor como esta requiere voluntad de reflexión y algún grado de humildad para reconocer posibles fallas en los juicios y las conductas propias, un proceso que frecuentemente es complejo, puesto que implica la necesidad de cambio personal y organizacional (Nguyen \& Schüßler, 2013).

Es recomendable que exista comunicación y retroalimentación del proceso de presupuesto de capital con un equipo en el que las directivas reciban puntos de vista autónomos, tanto de colaboradores internos como de accionistas y auditores externos, en el marco de un robusto gobierno corporativo. Esta es una tarea en la cual una junta directiva profesional, activa e independiente puede jugar un papel decisivo. Igualmente, se requiere implementar sistemas de evaluación y de compensación con base en los re- 
sultados de mediano y largo plazo (Axelsson, Jakovicka, \& Keddache, 2002; Nguyen, \& Schüßler, 2013).

\section{CONCLUSIONES}

El enfoque tradicional del presupuesto de capital, basado en los supuestos de la teoría neoclásica, se apoya en herramientas como el valor presente neto (VPN) y la tasa interna de retorno (TIR) para evaluar la viabilidad financiera de proyectos de inversión en activos fijos. Este tipo de decisiones corporativas se basa en la proyección de los flujos de caja que tratan de reflejar el comportamiento esperado de variables que no están bajo el control total de la empresa. Aunque la teoría financiera tradicional incorpora elementos como el riesgo y la incertidumbre, ella asume típicamente una perfecta previsión, un perfecto uso de la información y la ausencia de variables emocionales, influencias del entorno o errores cognitivos. A pesar del gran desarrollo conceptual y tecnológico de los métodos cuantitativos, es necesario reconocer que incluso el más detallado cálculo de indicadores financieros de evaluación de proyectos está sujeto a errores y es incapaz de eliminar la incertidumbre sobre el futuro. Las FCBC ofrecen un nuevo enfoque en el que los diferentes fenómenos psicológicos que afec- tan la toma de decisiones financieras (sesgos, heurísticos y efectos de encuadre) son incorporados explícitamente en el estudio de la realidad empresarial.

La evidencia mostrada en el presente artículo incorpora los más importantes estudios realizados sobre la influencia de los sesgos de optimismo y exceso de confianza en las decisiones de presupuesto de capital. Ella parece indicar que tales tendencias son sistemáticas y tienen un importante impacto en la asignación de recursos y en la creación de valor.

Puede afirmarse que la eliminación de los sesgos en el comportamiento es a la vez imposible e indeseable. Tal vez lo más conveniente sea que la empresa, empezando por sus altas directivas, reconozca la existencia de influencias del comportamiento en sus decisiones financieras, identifique sus impactos positivos y negativos e introduzca estos elementos explícitamente en su toma de decisiones, de forma que pueda aumentar la creación de valor para los accionistas y en general para la sociedad.

La influencia de elementos del comportamiento en el proceso de presupuesto de capital es un área relativamente nueva de estudio que ofrece amplias oportunidades de investigación. Son escasos los traba- 
jos en esta materia aplicados a casos latinoamericanos $\mathrm{y}$, en particular, a empresas pequeñas, que contemplen variables como la cultura, la edad o el género.

Las FCBC han estudiado apenas una pequeña parte del amplio conjunto de tendencias subjetivas que a la fecha han sido reconocidas. Será trascendental para el desarrollo de este campo que se conformen equipos interdisciplinarios en los que los aportes de psicólogos, sociólogos y antropólogos, entre otros profesionales, enriquezcan la comprensión que del comportamiento organizacional tienen los profesionales en ciencias económicas y administrativas.

\section{REFERENCIAS}

Adler, D. E. (2004). A behavioral theory of corporate finance. Strategy + Business, 34, 4-5.

Axelsson, H., Jakovicka, J., \& Keddache, M. (2002). Capital budgeting sophistication and performance: A puzzling relationship. Tesis de Maestría en Economía Financiera, Graduate Business School, Gotemburgo: Göteborg University.

Baker, M., \& Wurgler, J. (2013). Behavioral corporate finance: An updated survey. En G. M. Constantinides, M. Harris \& R.
M. Stulz (Eds.), Handbook of the Economics of Finance (Vol. 2, Part A, pp. 357-424). Amsterdam - Boston: Elsevier/NorthHolland.

Ben-David, I., Graham, J. R., \& Harvey, C. (2010). Managerial Miscalibration. Working Paper Series, Durham: Duke University.

Biondi, Y., \& Marzo, G. (2011). Decision making using behavioral finance for capital budgeting. En H. K. Baker \& P. English (Eds.), Capital budgeting valuation: Financial analysis for today'sinvestment projects (pp. 421-444). Hoboken: John Wiley $\&$ Sons.

Brealey, R. A., Myers, S. C., \& Allen, F. (2011). Principles of corporate finance $\left(10^{\mathrm{a}} \mathrm{ed}\right.$.). NuevaYork: McGraw-Hill/Irwin.

Burns, R. M., \& Walker, J. (1997). Capital budgeting techniques among the Fortune 500: A rationale approach. Managerial Finance, 23(9), 3-15.

Camerer, C., \& Lovallo, D. (1999). Overconfidence and excess entry: An experimental approach. The American Economic Review, 89(1), 306-318.

Crooks, E. (2010). BP: The Inside Story. Financial Times. Recuperado de www.ft.com.

Damasio, A. (1994), Descartes' error: Emotion, reason, and the 
human brain. Nueva York: Harper Collins Books.

Daniel, K., Hirshleifer, D., \& Subrahmanyam, A. (1998), A theory of overconfidence, selfattribution, and security market under- and over-reactions. Journal of Finance, 53, 1839-1885.

Dean, J. (1951), Capital budgeting. Nueva York: Columbia University Press.

Ehrhardt, M., \& Brigham, E. F. (2011). Corporate finance: A focused approach (4a ed.). Mason: South-Western/CENGAGE Learning.

Fairchild, R. (2004). Behavioral finance in a principal-agent model of capital budgeting. Working Paper Series. Bath: School of Management, University of Bath.

Fairchild, R. (2009). From behavioural to emotional corporate finance: A new research direction. Working Paper Series. Bath: School of Management, University of Bath.

Felleg, R., Moers, F., \& Renders, A. (2012). Investor reaction to higher earnings management incentives of overoptimistic CEOs. Working Paper. Maastricht: Department of Accounting and Information Management, Maastricht University.

Fornero, R. A. (2010). Las prácticas en las decisiones de inversión: sus características según los estudios empíricos. Working Paper. Córdoba, Argentina: XXX Jornadas Nacionales de Administración Financiera, Sociedad Argentina de Docentes en Administración Financiera SADAF.

Fox, J. (2010). The myth of the rational market: A history of risk, reward, and delusion on Wall Street. Nueva York: Harper Collins Publishers.

Gervais, S., \& Odean, T. (2001). Learning to be overconfident. Review of Financial Studies, 14(1), 1-27.

Gervais, S., Heaton, J. B., \& Odean, T. (2002). The positive role of overconfidence and optimism in investment policy. Working Paper. Pennsylvania: The Rodney L. White Center for Financial Research, The Wharton School, University of Pennsylvania.

Gervais, S., Heaton, J. B., \& Odean, T. (2011). Overconfidence, compensation contracts, and capital budgeting. Journal of Finance, 66(5), 1735-1777.

Gervais, S. (2010). Capital budgeting and other investment decisions. En H. K. Baker \& J. R. Nofsinger (Eds.), Behavioral finance: Investors, corporations, and markets (pp. 413-434). Hoboken: Wiley.

Graham, J. R., \& Harvey, C. R. (2001). The theory and practice 
of corporate finance: Evidence from the field. Journal of $\mathrm{Fi}$ nancial Economics, 60(2-3), 187-243.

Harris, E.P., Emmanuel, C. R., \& Komakech, S. (2009). Managerial judgment and strategic investment decisions: A crosssectional survey. Oxford: Elsevier.

Heaton, J. B. (2002). Managerial optimism and corporate finance. Financial Management, 31(2), 33-45.

Hribar, P., \& Yang, H. (2012). CEO overconfidence and management forecasting. Working Paper. Iowa: University of Iowa-Cornell University.

Hribar, P., \& Yang, H. (2010). Does CEO overconfidence affect management forecasting and subsequent earnings management? Iowa: Working Paper, University of Iowa-Cornell University.

Koellinger, P., Minniti, M., \& Schade, C. (2007). I think I can, I think I can: Overconfidence and entrepreneurial behavior. Journal of Economic Psychology, 28(4), 502-527.

Kida, T., Moreno, K., \& Smith, J. E. (2001). The influence of affect on managers. Capital-budgeting decisions. Contemporary Accounting Research, 18(3), 477-494.

Krauze, R. (1990), La filosofia de Antonio Caso. México: Univer- sidad Nacional Autónoma de México.

Landier, A., \& Thesmar, D. (2009). Financial contracting with optimistic entrepreneurs. Review of Financial Studies, 22(1), 117-150. Laux, J. (2011). Topics in finance. Part VI .Capital Budgeting. American Journal of Business Education, 4, 29-37.

LeDoux, J. E. (1993), Emotional memory systems in the brain. Behavioral and Brain Research, 58, 69-79.

LeDoux, J. E. (1996). The emotional brain: The mysterious underpinnings of emotional life, Nueva York: Simon \& Schuster. Malmendier, U. \& Tate, G. A. (2005). Does overconfidence affect corporate investment? CEO overconfidence measures revisited. European Financial Management, 11(5), 649-659.

Mattson, L., \& Weibull, J. (2002). Probabilistic choice and procedurally bounded rationality. Games and Economic Behavior, 41, 61-78.

McDonald, R. L. (1998). Real options and rules of thumb in capital budgeting. Working Paper Series. Evanston: Finance Department, Kellogg School, Northwestern University.

Nguyen, T., \& Schüßler, A. (2013). How to make better decisions? Lessons learned from behavior- 
al corporate finance. International Business Research, 6(1), 187-198.

Pohlman, R. A., Santiago, E. S., \& Markel, F. L. (1988). Cash flow estimation practices of large firms. Financial Management, 17(2), 71-79.

Real Academia Española (RAE) (2013). Diccionario de la Lengua Española (XXII ed.). Disponible en www.rae.es

Ross, S. A. (1995). Uses, abuses and alternatives to the net present value. Financial Management, 24(3), 96-102.

Sautner, Z., \& Weber, M. (2009). How do managers behave in stock option plans? Clinical evidence from exercise and survey data. Journal of Financial Research, 32(2), 123-155.

Scarpetta, S., Hemmings, P., Tressel, T., \& Woo, J. (2002). The role of policy and institutions for productivity and firm dynamics: Evidence from micro and industry data. Working Paper No. 329. OECD Economics Department.

Schoar, A., \& Bertrand, M. (2003). Managing with style: The effect of managers on firm policies. The Quarterly Journal of Economics, 118(4), 1169-1208.

Schönbohm, A., \& Zahn, A. (2012). Corporate capital budgeting: Success factors from a behavioral perspective. Research Report $\mathrm{n}^{\circ}$ 21. Kaiserslautern: Technische Universität Kaiserslautern.

Shah, P. (2013). Behavioral corporate finance: A new paradigm shift to understand corporate decisions. Global Research Analysis, 2, 85-86.

Shefrin, H., \& Cervellati, E. M. (2011). BP's failure to debias: Underscoring the importance of behavioral corporate finance. Working Paper. Santa Clara: Leavy School of Business, Santa Clara University.

Statman, M., \& Caldwlell, D. (1987). Applying behavioral finance to capital budgeting: project terminations. Financial Management, 16(4), 7-15.

Statman, M. (2008). Countries and cultures in behavioral finance. CFA Institute Conference. Proceedings Quarterly, 25(3), 3844.

Stein, J. C. (1996). Rational capital budgeting in an irrational world. Working Paper 5496, NBER.

Stowe, J., \& Gagne, J. R. (2012). Capital budgeting. En M. R. Clayman, M. S. Fridson \& G. $\mathrm{H}$. Troughton (Eds.), Corporate Finance: A Practical Approach ( $2^{\text {a }}$ ed., pp. 47-126). Hoboken: John Wiley \& Sons.

Svenson, O. (1981). Are we all less risky and more skillful than our fellow drivers? Acta Psychologica, 47(2), 143-148. 
Terry,E.(2010). Theimpact of scena- Weinstein, N. D. (1980). Unrealisrio presentation on capital budgeting decisions. Working Paper. Toronto: Ted Rogers School of Business Management, Ryerson tic optimism about future life events. Journal of Personality and Social Psychology, 39(5), 806-820.

University. 